\title{
Subunit composition of native ATP-sensitive K+ channels in mouse portal vein smooth muscle
}

\author{
Tadashi Yamamoto ${ }^{1,2}$, Kohei Takahara ${ }^{1}$, Keiichiro Uchida ${ }^{1}$, Noriyoshi Teramoto ${ }^{1,2}$ \\ ${ }^{I}$ Dept. Pharmacol., Fac. Med., Saga Univ., Japan, ${ }^{2}$ Dept. Biomed. Eng., Grad. Sch. Biomed. Eng., Tohoku Univ., Japan
}

Molecular biology analyses and immunohistochemistry experiments were performed to investigate the main subunits of native ATP-sensitive $\mathrm{K}+$ channels (KATP channels) that colocalize in mouse portal vein. In portal veins from three different mouse strains (BALB/c, C57BL/6 and ICR mice), real-time PCR revealed that the expression of the gene encoding Kir6.1 was greater than that of the gene encoding Kir6.2 and that the gene encoding SUR2B was more highly expressed than those encoding SUR1 and SUR2A. Thus, the predominant KATP channel subunits expressed in mouse portal vein are likely to be Kir6.1 as the channel pore and SUR2B as the modulatory sulphonylurea receptor (SUR). In transverse sections of the mouse portal vein, an in situ proximity ligation assay (PLA) using primary antibodies against Kir6.1 and SUR2B detected green 'hot spots' of immunofluorescence, suggesting that Kir6.1 and SUR2B subunit proteins were closely colocalized in the vascular smooth muscle layers. Similarly, PLA also detected immunofluorescence signals in single smooth muscle cells freshly dispersed from the mouse portal vein, indicating colocalization of Kir6.1 and SUR2B subunit proteins. These findings provide strong evidence that native KATP channels in mouse portal vein myocytes are composed mainly of Kir6.1 and SUR2B subunits (i.e. Kir6.1/SUR2B channels). 